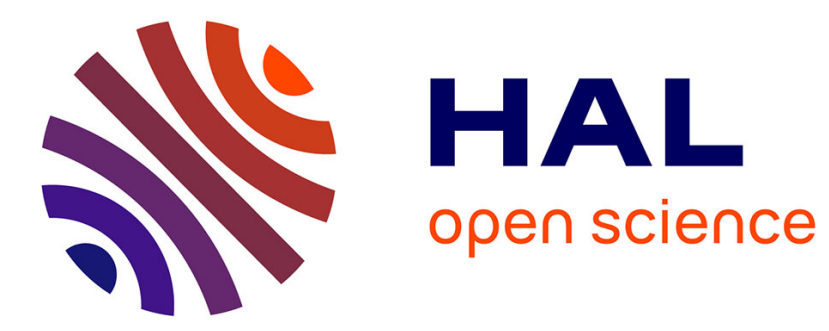

\title{
Spatialisation de la demande en eau d'irrigation estimée par un modèle bioéconomique
}

Pierre-Alain Jayet, Delphine Barberis, Pierre Humblot, Anna Lungarska

\section{To cite this version:}

Pierre-Alain Jayet, Delphine Barberis, Pierre Humblot, Anna Lungarska. Spatialisation de la demande en eau d'irrigation estimée par un modèle bioéconomique. Revue Internationale de Géomatique, 2018, 28 (4), pp.485-503. 10.3166/rig.2018.00064 . hal-02617894

\section{HAL Id: hal-02617894 \\ https://hal.inrae.fr/hal-02617894}

Submitted on 25 May 2020

HAL is a multi-disciplinary open access archive for the deposit and dissemination of scientific research documents, whether they are published or not. The documents may come from teaching and research institutions in France or abroad, or from public or private research centers.
L'archive ouverte pluridisciplinaire HAL, est destinée au dépôt et à la diffusion de documents scientifiques de niveau recherche, publiés ou non, émanant des établissements d'enseignement et de recherche français ou étrangers, des laboratoires publics ou privés.

\section{(ㄷ)(1) $\$$}

Distributed under a Creative Commons Attribution - NonCommerciall 4.0 International 


\title{
Spatialisation de la demande en eau agricole en France par l'intégration de l'eau d'irrigation dans un modèle bioéconomique
}

\author{
Pierre-Alain Jayet ${ }^{1}$, Delphine Barberis ${ }^{1}$, Pierre Humblot ${ }^{1}$, Anna \\ Lungarska ${ }^{1}$
}

\author{
1. UMR INRA-Agro Paris Tech "Economie Publique” \\ 1 Avenue Lucien Brétignières, 78850 Thiverval-Grignon, France \\ pierre-alain.jayet@inra.fr
}

\begin{abstract}
RÉSUMÉ. Les pressions actuelles exercées sur la ressource en eau et la menace potentielle du changement climatique devraient fortement influencer sa disponibilité et, de façon corrélative, la demande d'irrigation. Nous développons dans cet article une approche centrée sur l'eau comme facteur de production agricole et sur la demande française en eau d'irrigation. La méthode repose sur le couplage du modèle d'offre agricole AROPAj avec le modèle de culture STICS ainsi que sur l'obtention de cartes à haute résolution. Nous proposons d'illustrer la capacité du modèle à enrichir l'analyse spatiale de la demande d'irrigation et des disparités régionales. Focalisée sur les principales de cultures de vente, l'étude montre l'importance des cultures très consommatrices d'eau d'irrigation dans les zones fortement irriguées à l'ouest et au sud-est de la France. Elle montre également dans quelle mesure le modèle pourra traiter de l'importance que pourrait prendre l'irrigation dans d'autres régions dans un contexte de changement climatique prononcé.
\end{abstract}

ABSTRACT. Present pressures on the water resource and the potential threat of climate change should strongly impact water availability and, consequently, irrigation demand. In order to help public decision-makers, we develop an approach where water is modeled as an input, and assess water demand in France. The method combines the economic model AROPAj, the STICS crop model, and the generation of high-resolution maps. As an illustration of the possible outputs of our modeling approach, we propose an application based on the spatial analysis of irrigation demand and regional disparities. This study reveals highly irrigated areas in the west and south-east of France, explained by the establishment of water-intensive crops like maize and sunflower. More importantly, it highlights the model capacity to enlighten the complex linkage between climate change and the demand for irrigation water even in regions where crops are not strongly water-intensive.

MOTS-CLÉS : demande en eau d'irrigation, modèle bioéconomique d'offre agricole, modèle de culture, fonction de rendement, occupation du sol

KEYWORDS: agricultural water demand, bio-economic supply model, crop model, yield function, land use 


\section{Introduction}

Avec 3 milliards de mètres cubes prélevés en 2009, l'agriculture française est un secteur d'activité très consommateur d'eau (Eau France, 2012). Face à une demande alimentaire croissante corrélée à une intensification des facteurs de production et une augmentation des terres cultivées irriguées, la menace d'une potentielle baisse de la disponibilité en eau est plus que jamais présente, accentuée par les conséquences probables du changement climatique. En France, l'existence de pratiques d'irrigation disparates d'une région à l'autre (intensives dans le Sud et moins marquées dans le Nord) complexifie les prises de décision territoriale en matière de gestion intégrée de la ressource. Les tensions actuelles s'intensifient par le risque d'augmentation des restrictions de prélèvements et d'usages de l'eau. Dans un objectif d'aide à la décision publique, la mise en place d'outils de modélisation appropriés apparaît indispensable à une gestion optimale de la ressource en accord avec le maintien de hauts niveaux de production agricole. Au vu d'un environnement économique et physique mondial en constante évolution, il convient d'évaluer et plus encore de spatialiser la demande d'irrigation actuelle afin d'en faire émerger d'éventuelles stratégies d'adaptation.

Dans cette étude, nous partons du monde agricole tel que représenté par le modèle agro-économique, AROPAj, et proposons une analyse prospective centrée sur les liens entre rendements et intrants fournis par le modèle de culture STICS. Nous optons ainsi pour l'emploi d'un modèle "bioéconomique » ayant pour principale visée la simulation du comportement des agriculteurs par incorporation d'informations agronomiques (Janssen et van Ittersum, 2007). Il s'agit, en d'autres termes, d'analyser les choix des producteurs quant à l'assolement et l'utilisation d'intrants mais également d'anticiper les externalités associées. Cette forme d'approche est la plus fréquemment utilisée dans l'étude des enjeux environnementaux et des intrants agricoles, comme en atteste la littérature (Cortignani et Severini, 2009 ; Kampas et al., 2012 ; Medellín-Azuara et al., 2012 ; Jayet et Petsakos, 2013 ; Graveline et Mérel, 2014 ; Larson et al., 1996 ; Godard et al., 2008). Des fonctions de réponse d'intrants (eau et azote) sont déterminées pour chacune des 8 cultures les plus commercialisées en Europe. De multiples informations telles que la marge brute et la demande d'irrigation sont ainsi obtenues à l'échelle du pays. Bien que l'approche soit conçue au niveau européen, la France est privilégiée en raison de sa représentativité en termes de diversité de productions agricoles, de climat et de sol.

L'intégration du facteur eau corrélée au suivi de la fertilisation azotée, et la simulation d'une grande variété de productions à l'échelle d'un pays entier sont autant d'éléments qui font la force de la démarche développée. Cette étude constitue une extension des travaux réalisés par Humblot et al. (2017). Une attention particulière est accordée à la spatialisation des sorties du modèle et plus particulièrement au prolongement de la méthode de Cantelaube et al. (2012) à la nouvelle dimension de l'eau. L'obtention de cartes à haute résolution permettant de 
visualiser la disparité des pratiques d'irrigation d'une région à l'autre est un élément clé de la démarche proposée.

Dans la section suivante, nous décrivons la méthode développée et plus précisément les éléments du couplage AROPAj-STICS, la problématique d'optimisation associée, l'élaboration des fonctions de rendement et la procédure de désagrégation spatiale des sorties du modèle. Dans un deuxième temps, nous illustrons la capacité offerte par le modèle à l'analyse de la demande d'irrigation en France et de son hétérogénéité inter et intra régionale. Nous concluons sur les perspectives offertes par la démarche engagée.

\section{Matériel et méthode : adaptation d'un modèle bioéconomique et spatialisation des résultats}

Nous partons du monde agricole tel que représenté par le modèle agroéconomique AROPAj. Nous proposons une analyse centrée sur les liens entre les rendements des cultures et deux des principaux intrants que sont l'eau et l'azote. Ces liens sont fournis par le modèle de culture STICS (Godard et al. 2008 ; Leclère et al. 2013 ; Humblot et al. 2017) (figure 1), selon une présentation succincte faite en sections 2.1 et 2.2. La désagrégation des sorties du modèle à l'échelle du pixel pour l'obtention de représentations cartographiques à haute résolution est rendue possible par l'ajout d'un module complémentaire de spatialisation (cf. section 2.3).

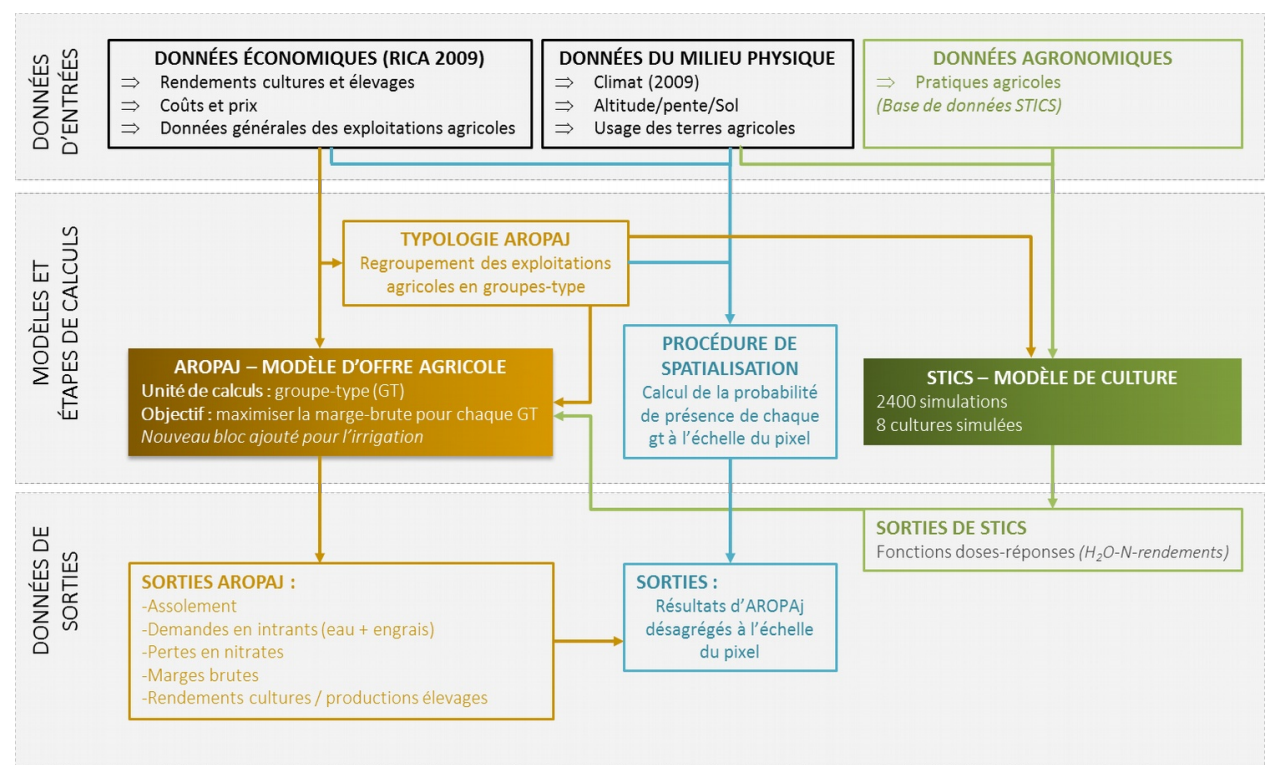

FIGURE 1. Méthode générale et couplage des modèles AROPAj-STICS

En jaune: module AROPAj; vert: module STICS ; bleu : module de spatialisation des résultats 


\subsection{Données et outils de modélisation}

\subsubsection{Le modèle d'offre agricole $A R O P A j$}

AROPAj est un modèle d'offre agricole de programmation linéaire de type mono-périodique et statique ${ }^{1}$. Il s'appuie sur l'emploi des données du Réseau d'Information Comptable Agricole (RICA) et sur la conception de groupe-types agricoles statistiquement représentatifs visant à capturer la diversité des exploitations de l'Union européenne. L'hypothèse économique qui le sous-tend est que chaque groupe-type maximise sa marge brute sous différentes contraintes techniques (rotations agricoles, cheptel et terre disponible, alimentation du bétail) et économiques (avec les règles de la Politique Agricole Commune - PAC). Ces groupes-types (GT) ou agents économiques sont obtenus suite à l'agrégation, par classification automatique, de fermes aux caractéristiques proches, extraites du RICA. L'année d'observation utilisée dans cet article est 2009. Les 4 critères utilisés pour la classification sont: l'orientation technico-économique des exploitations (normalisation RICA en 14 types, dont 12 utilisés par AROPAj), la part de surfaces irriguées, la dimension économique (normalisation RICA en 9 catégories) et l'altitude ( 3 classes). Cette typologie conduisant à représenter 350000 exploitants (à temps complet, dans le RICA) par 160 GTs est la plus « fine» qui soit compatible avec le respect des règles de confidentialité propres à l'accès aux données du RICA et avec la qualité statistique des estimations des paramètres technico-économiques du modèle. Elle présente l'avantage de réduire considérablement le temps de calcul numériques des simulations qui font appel aux solveurs traitant des problèmes d'optimisation.

Le périmètre du modèle AROPAj est associé aux principales productions animales et végétales à l'exception de l'horticulture, de la viticulture et de l'arboriculture. Les différents modules (représentation technico-économiques des systèmes de production et couplage AROPAj-STICS) sont d'abord mobilisés pour produire de l'information brute (e.g. caractéristiques des exploitations en termes de charges variables et de rendements). L'exploitation du modèle conduit ensuite à estimer le partage optimal des terres entre activités agricoles, les quantités produites et les ressources consommées, les pollutions associées (gaz à effet de serre, pollutions azotées) dans le cadre de scénarios (climatiques, économiques). Ces résultats sont produits à différentes échelles standard avec l'exploitation agricole type, la région, l'Etat membre et l'Union Européenne, et avec un haut niveau de résolution spatiale en mobilisant le module de «spatialisation » (figure 1).

La force de l'outil est sa généricité et plus spécifiquement sa capacité à rendre compte avec précision du comportement d'un grand nombre d'exploitations à travers l'étude de divers mécanismes d'adaptation (changement d'allocation des sols, variation des quantités d'intrants, ajustement du capital animal). La version de base d'AROPAj ne prend pas en compte des différents aspects techniques et physiques des pratiques agricoles. Le couplage à un modèle de culture permet

Jayet, P.-A. et al. (n.d.). The European agro-economic AROPAj model: https://www6.versailles-grignon.inra.fr/economie publique/Publications 
l'intégration de paramètres agronomiques propres à raffiner la relation entre intrants et rendements qui deviennent des variables endogènes du modèle. Il est alors possible d'affiner la simulation des systèmes agricoles en réponse au changement climatique et au changement de l'environnement économique (prix, PAC).

\subsubsection{Le modèle de culture STICS}

Quoique plus performant dans les zones tempérées, STICS est un modèle générique capable de simuler la croissance d'un grand nombre de cultures dans n'importe quelle région du monde. Il modélise les processus variés se déroulant dans chaque partie d'une plante et fonctionne à un pas de temps journalier, sur une année calendaire voire deux suivant le type de production considérée (Brisson et al., 2003). Outre le fait de pouvoir disposer du code, STICS a l'avantage d'être l'un des modèles de culture les plus cités dans le monde académique (Keating et Thorburn, 2016).

Divers éléments sont requis en entrée de STICS (figure 1) :

- Des paramètres climatiques fournies par un modèle atmosphérique (e.g. ARPEGE) utilisé pour la prévision numérique des conditions météorologiques (Déqué et al., 1994)

- Des informations pédologiques provenant de la base de données européennes des sols (ESDB)

- Des renseignements liés aux pratiques agricoles issues de la base interne de STICS

De multiples variables agronomiques et environnementales (rendements, pertes d'azote, quantités d'engrais et volume d'eau requis sous objectif d'optimisation du profit) sont estimées en sortie du modèle. Ces résultats contribuent à l'élaboration d'un jeu de fonctions dose-réponse, alliant rendements et intrants -eau et azotepour chacune des principales cultures en production chez chacun des GT.

\subsection{Intégration du facteur eau : obtention de fonctions dose-réponse à 2 variables}

TABLE 1. Récapitulatif des différentes combinaisons modélisées lors de la phase de création des fonctions dose-réponse dans STICS

\begin{tabular}{cc}
\hline Options de saisie de données & Nombre de modalités par culture \\
\hline Climat & 1 \\
Sol & 10 \\
Variétés culturales* & 16 \\
Date de semis* & 5 \\
Précédent cultural & 1 \\
Classe d'altitude & 3 \\
\hline Nombre total de combinaisons & $\mathbf{2 4 0 0}$ \\
\hline *Dépend du type de culture considérée, ces deux options créent un \\
total de trois combinaisons \\
Source : Humblot et al., 2017
\end{tabular}


En pratique, il existe peu d'information à l'échelle d'une ferme virtuelle susceptible d'alimenter STICS (e.g. le type de sol et la variété culturale sont inconnus). Pour pallier cette difficulté, ce sont en général un total de 2400 simulations STICS qui sont réalisées selon différentes modalités de culture (type de sol, date de semis, variété) (tableau 1) pour chaque GT et pour chacune des 9 principales productions commercialisées en Europe (blé tendre, blé dur, maïs, orge, colza, tournesol, betterave, pomme de terre, soja).

Pour les systèmes agricoles non irrigués, l'apport en eau est purement exogène, c'est-à-dire dépendant des conditions météorologiques. Dans le cas contraire, cette variable est modulable dans la mesure où elle devient une " variable de commande » du système de production. Dans tous les cas, il s'agit de construire des fonctions de rendement intégrant l'eau et l'azote comme intrants à disposition de l'agent économique. En cas de changement climatique ou économique, l'eau d'irrigation peut émerger ou disparaître chez certains systèmes de productions, et c'est ce que le modèle cherche à appréhender, même si l'irrigation sera toujours contrainte par des investissements qui eux ne relèvent pas du modèle.

Le processus est conduit en deux étapes afin d'obtenir les rendements STICS pour différents volumes d'eau apportés. Une première simulation est réalisée avec des entrées en azote non limitantes (se reporter à la méthode développée par Godard (2008) pour plus d'informations concernant cet intrant). Les quantités d'eau et dates de passage sont fixées automatiquement par STICS. Les apports sont ainsi ajustés aux besoins de la culture et aux données climatiques. Dans un deuxième temps, l'option d'irrigation automatique du modèle est désactivée. Les informations de la première phase sont stockées. Pour des dates de passage identiques à l'étape précédente, des simulations STICS successives sont effectuées pour des volumes d'irrigation réduits progressivement d'un facteur compris entre 0 et $100 \%$ $(0 / 20 / 40 / 60 / 80 / 100 \%)$ (figure 2). De cette manière, il est possible d'imposer un stress hydrique. Ce dernier affecte les rendements de façon plus ou moins importante selon le facteur de réduction fixé.

En conséquence, de multiples relations $\mathrm{N}-\mathrm{H}_{2} \mathrm{O}$-rendement sont obtenues et prennent la forme d'une fonction de type Mitscherlich-Baule. La transcription graphique de la relation mathématique proposée conduit à représenter une surface (figure 3). La méthode a été développée et illustrée par Humblot et al. (2017). Les fonctions de rendement sont calibrées sur la base d'une forme fonctionnelle de type exponentielle dont les paramètres sont un potentiel agronomique (l'asymptote), des termes de courbure (associées à une forme d'efficience) et des apports naturels d'eau et d'azote :

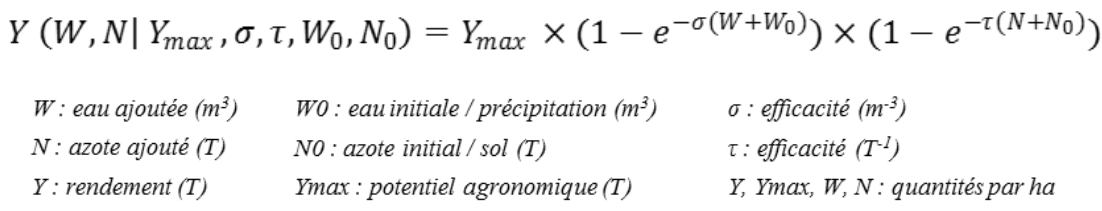




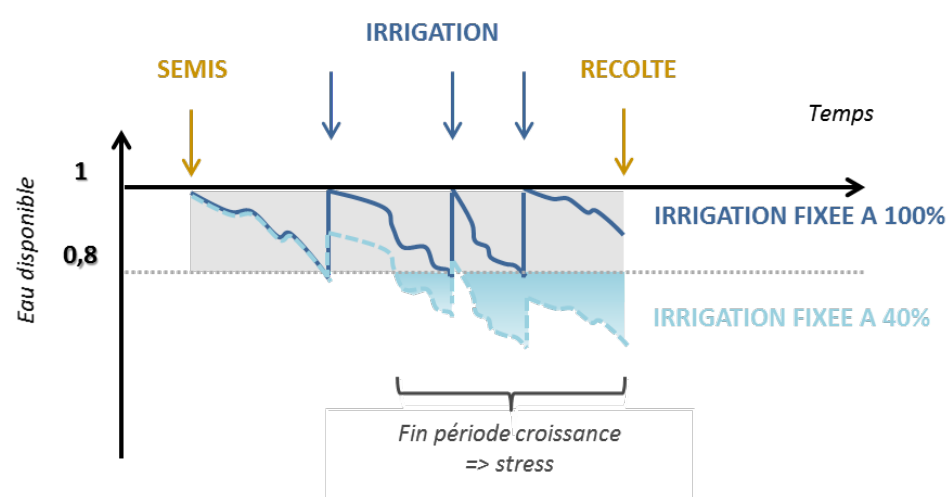

FIGURE 2. Simulation de l'impact des apports d'eau sur les rendements (bleu foncé : activation de l'option STICS d'irrigation automatique [apports fixés à 100\%] ; bleu clair : quantité d'irrigation réduite mais passages effectués aux mêmes dates (dans cet exemple, apports fixés à $40 \%$ par rapport à l'étape précédente)

Pour chaque couple « groupe type / culture », le processus d'élaboration de la fonction de rendement est conduit en 2 étapes : (i) en sélectionnant les surfaces « $\mathrm{H}_{2} \mathrm{O}-\mathrm{N}$-rendement » calibrées à partir des simulations STICS réalisées selon les jeux de paramètres présentés dans le tableau 1 selon un critère d'éligibilité associé au rendement observé ; (ii) parmi les surfaces éligibles, en retenant celle qui optimise un critère économique fondé sur des rapports de prix (Humblot et al., 2017). Ce critère est un " angle» entre un vecteur orthogonal à la surface et un vecteur de prix satisfaisant l'hypothèse de maximisation de la marge brute par unité de surface pour le rendement observé. La meilleure fonction est celle qui minimise l'angle entre les deux vecteurs (figure 3).

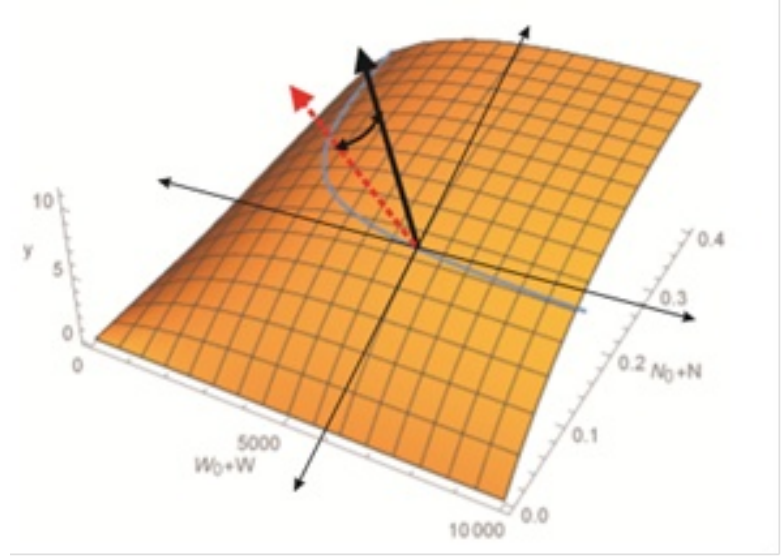

FIGURE 3. Surface de rendement en réponse aux intrants eau et azote :

Représentation graphique du vecteur de prix et du vecteur " gradient " perpendiculaire au plan tangent à la surface de production, pour un niveau de rendement observé. Courbe bleue : isoquante associée au rendement observé. Source : Humblot et al., 2017. 
Une fois calibrées, les fonctions de rendement sont introduites en entrée du modèle d'offre agricole (figure 1), traduisant ainsi le couplage AROPAj-STICS. La demande en eau d'irrigation est évaluée en sortie de simulation, dans un contexte de maximisation de la marge brute des exploitations agricoles, indépendamment d'une offre qui peut s'avérer limitante. Les résultats finaux sont désagrégés spatialement dans le but d'obtenir des représentations cartographiques à haute résolution.

\subsection{Méthode de désagrégation spatiale des sorties du modèle à une échelle fine}

En raison de la politique de confidentialité du RICA, l'exploitation de résultats utilisant le positionnement géographique d'un individu est prohibée. A ce stade, la seule information disponible concerne l'appartenance d'un GT d'AROPAj à une région spécifique. Cependant, il est possible de localiser de façon probabiliste la présence des GT d'une région à l'échelle du pixel (et de façon pragmatique à l'échelle du polygone) en mobilisant des méthodes d'économétrie spatiale et des données physiques complémentaires. Il s'agit dans un premier temps « d'expliquer » la présence des différentes activités agricoles en fonction des caractéristiques physiques locales. Dans un second temps, on calcule la contribution de chacun des GT aux activités agricoles du pixel (ou du polygone). Il est alors possible de distribuer géographiquement toutes les sorties du modèle d'offre agricole avec un haut niveau de résolution spatiale.

La méthode a été développée par Chakir (2009) et mise en œuvre par Cantelaube et al. (2012) à l'échelle européenne pour une version antérieure du modèle AROPAj. Dans cette étude, la procédure de spatialisation a été étendue à la version V5 d'AROPAj associée au RICA de l'année 2009, avec la possibilité de tenir compte des parts de surface irriguée des GT utilisées pour la nouvelle typologie.

La figure 4 illustre l'ensemble des étapes du processus. De par l'apport des données régionales du RICA, une cartographie précise des GT est établie au sein de chaque région par mobilisation de divers paramètres propres au milieu physique et au domaine agronomique (occupation du sol, climat, sol, altitude). Dans le but d'initier la première phase de traitement, les différentes couches d'informations géoréférencées sont préparées puis intersectées (étapes 1 et 2 de la figure 4).

Un premier modèle économétrique ${ }^{2}$ permet d'estimer en chaque pixel la probabilité de présence de chacune des principales activités agricoles existant dans la base de données d'occupation du sol LUCAS ${ }^{3}$. L'estimation est réalisée pour chacune des régions du RICA, en fonction de variables explicatives extraites de diverses bases de données (CLC, MNT et MARS) (étapes 3 et 4 de la figure 4). Concernant plus spécifiquement l'élément « eau », les informations sont issues de la base LUCAS. Une grille régulière de $25 \times 25 \mathrm{~km}$ est ainsi définie. Cette dernière a l'avantage d'être déjà intersectée avec les autres couches utilisées (ex : climat). Pour

\footnotetext{
MNL model : multinomial logit model

LUCAS : Land Use/Cover Area frame Survey
} 
chaque maille de cette grille, la présence de points irrigués LUCAS est testée. La détection d'au moins un point dans une maille donnée signifie une probabilité plus forte d'abriter des GT irrigués.

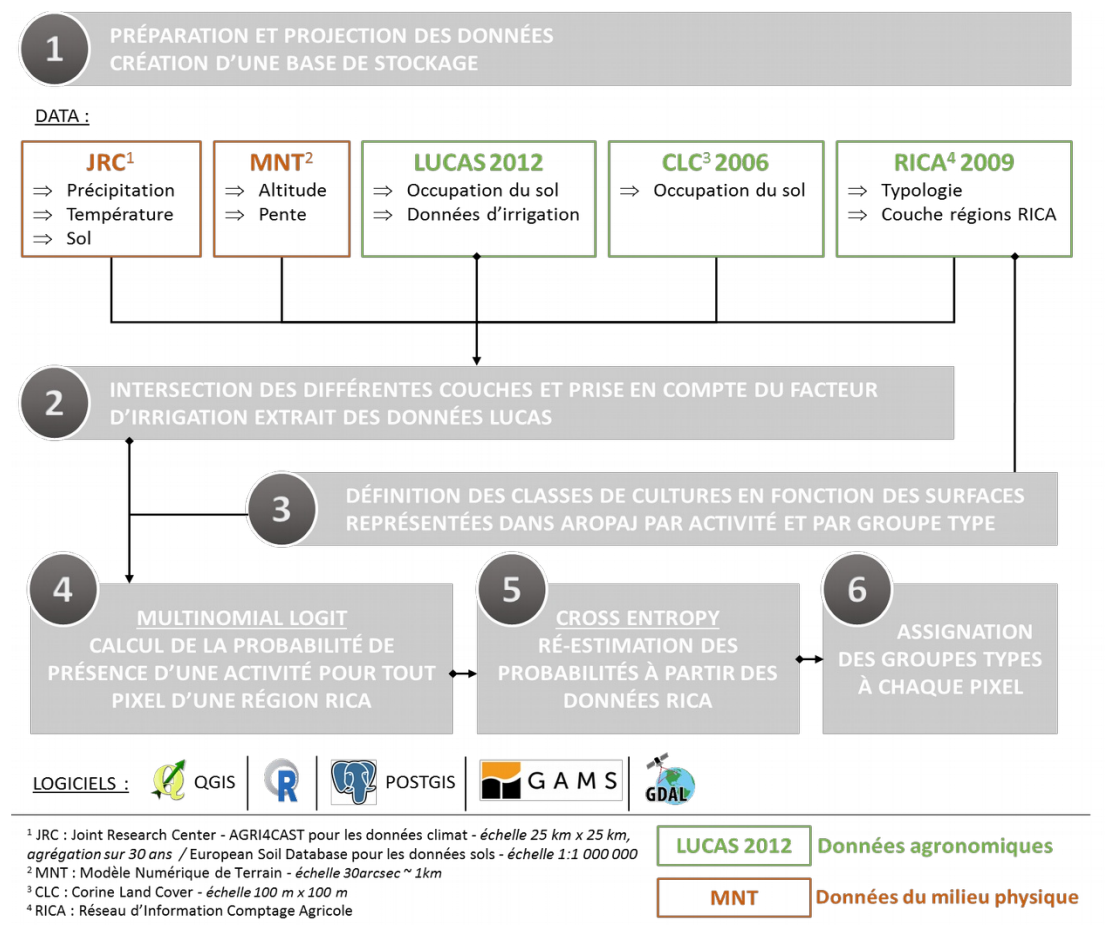

FIGURE 4. Méthode de calcul d'estimation de la localisation probabiliste des groupes types d'exploitations agricoles d'AROPAj à l'échelle du pixel.

A ce stade, les informations fournies par le RICA, telles que les proportions régionales d'allocation des terres allouées aux différentes cultures ne sont pas prises en compte. Les probabilités calculées lors de la phase précédente sont affinées par une méthode d'entropie croisée généralisée (Cantelaube et al., 2012), afin de minimiser la différence entre les parts d'utilisation des terres estimées par le modèle et celles observées par le RICA (étape 5 de la figure 4). Enfin, sous la contrainte que la somme des contributions des GT à l'activité agricole de la maille géographique (i.e. probabilités de présence) soit inférieure ou égale à 1 (égale à 1 en tenant compte des activités «non AROPAj »), on calcule la contribution de chaque GT sur chacune des mailles de la région (étape 6 de la figure 4).

Les sorties du modèle AROPAj (e.g. marge brute, demande en eau d'irrigation, pollution) sont alors distribuées en tenant compte des surfaces agricoles utiles des GTS et de leur probabilité de présence géographique. La mise en place d'algorithmes a permis l'automatisation des calculs pour les différentes phases de la 
spatialisation. Il en résulte l'obtention de cartes pour différentes échelles de restitution (parcelles, bassin versant, régions, pays).

\section{Validation et application de la méthode de spatialisation}

\subsection{Validation de la démarche sur un cas régional}

Afin de valider la méthode de désagrégation spatiale, nous comparons les surfaces des cultures estimées grâce aux méthodes «logit multinomial» et «entropie croisée généralisée » avec les surfaces déclarées dans le registre parcellaire graphique (RPG). Cette comparaison est faite pour la région PoitouCharente à partir des petites régions agricoles $(\mathrm{PRA})^{4}$, au nombre de 30 dans la région. Cette échelle a été choisie afin d'obtenir une vision plus générale sur la qualité de nos prédictions. Le découpage en PRA répond au besoin d'un découpage aussi homogène que possible au point de vue agricole, inspiré des régions géographiques tout en s'affranchissant des limites administratives. La taille des PRA est intermédiaire entre la commune et le département. Même si l'évolution de l'agriculture et la redistribution des activités remettent en question ce découpage, il est toujours utilisé dans les études portant sur le secteur agricole.

Pour mieux comprendre les résultats de la comparaison, il est nécessaire d'expliciter les caractéristiques et les limites du RPG. Un descriptif plus détaillé est proposé par Cantelaube et Carles (2014), en rappelant quelques points clés : (i) le RPG est obtenu en lien avec les aides de la PAC de l'UE ; (ii) les agriculteurs bénéficiaires sont tenus de préciser géographiquement les limites des parcelles qu'ils exploitent ainsi que les surfaces des différentes cultures présentent sur ces parcelles. Ces cultures sont regroupées en 28 classes. Selon Cantelaube et Carles (2014) il s'agit de la meilleure représentation de l'espace agricole français. Le RPG couvre approximativement $91,4 \%$ de la surface agricole totale (comprenant des surfaces non rattachées aux exploitations et qui ne sont pas couvertes par la PAC). La différence dans les surfaces RPG et les surfaces données par les statistiques nationales (Agreste) est néanmoins faible sauf pour les prairies $(11,8 \%$ en 2009 avec une sous-représentation dans le RPG).

Nous montrons de deux manières la qualité de la représentation spatiale des activités agricoles par le module de spatialisation associé au modèle AROPAj.

Les histogrammes de la figure 5 montrent que les différences entre les parts de surface des cultures dans les PRA sont relativement faibles pour le maïs, l'orge, le tournesol et le colza (majoritairement inférieures à $10 \%$ ). La prédiction est moins bonne pour le blé tendre où 14 PRA ont des différences supérieures à $10 \%$. Pour les prairies, les différences peuvent être attribuées à la déficience du RPG par rapport à cet usage des terres. Les friches sont moins bien prédites aussi alors que les surfaces non-AROPAj (NAU) ne sont pas comparables puisque pour la spatialisation elles

$4 \quad$ http://agreste.agriculture.gouv.fr/definitions/zonages/ 
intègrent des forêts qui ne relèvent pas nécessairement de la propriété d'exploitants agricoles couverts par la PAC et donc renseignés dans le RPG.
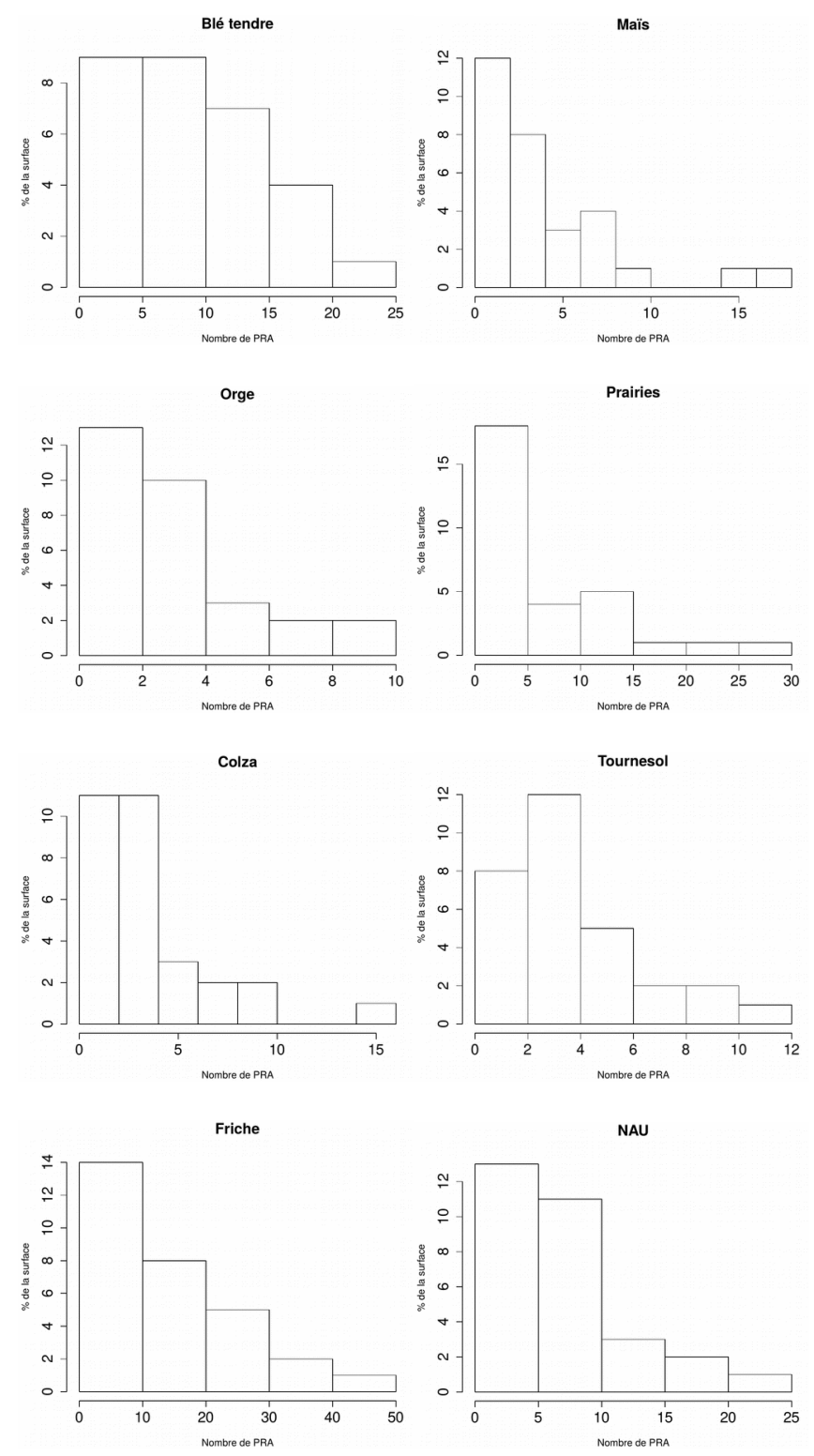

FIGURE 5. Histogrammes des différences absolues entre les parts de surface des classes de cultures dans les PRA selon les surfaces RPG et selon les surfaces estimées pour la spatialisation 
Les cartes de la figure 6 illustrent la distribution géographique de ces différences. Il apparaît en particulier que les surfaces en maïs, culture très intensive en eau, semblent bien réparties sur le territoire. Cela est important pour l'analyse développée dans cet article, puisque nous cherchons à donner une représentation fine de la demande en eau par l'agriculture.

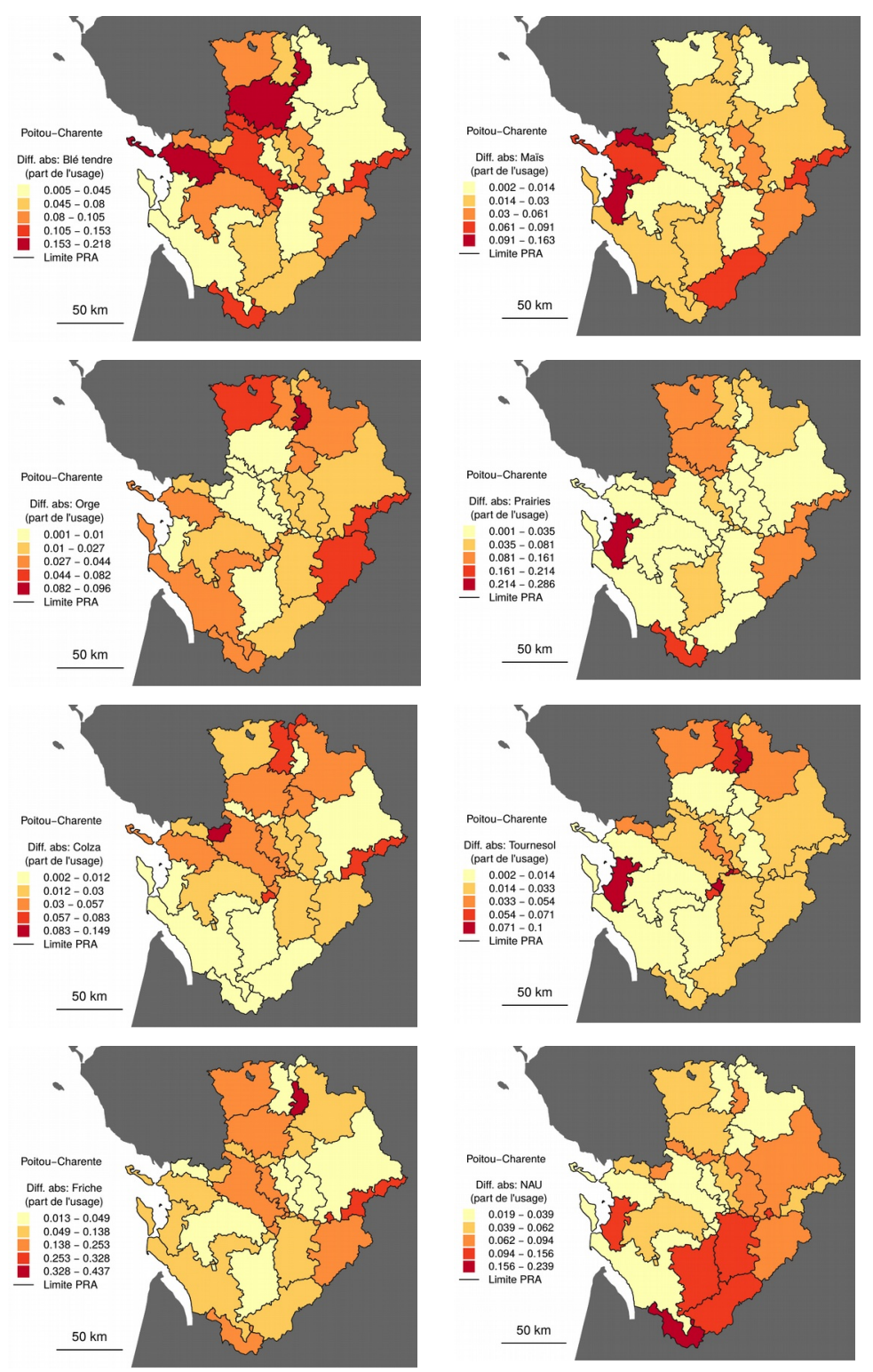

FIGURE 6. Différence absolue entre parts de surface prédites et fournies par le RPG 


\subsection{Illustration pour la demande en eau d'irrigation et sa disparité inter- régionale}

Parmi les applications valorisant la méthode de spatialisation, nous retenons ici la demande en eau agricole en 2009 , année de référence pour le calibrage d'AROPAj et pour laquelle nous conduisons une étude sur les impacts du changement climatique. A travers cet exemple, l'objectif est triple :

- Donner un aperçu des multiples sorties du modèle ;

- Mettre en évidence la capacité du couplage AROPAj-STICS à capter la diversité régionale des systèmes agricoles ;

- Etablir des analyses spatiales à différentes échelles (nationale ou régionale) dans le but d'illustrer la flexibilité du modèle :

Ce domaine d'étude a pour avantage d'aborder deux grandes thématiques largement traitées dans la littérature : l'eau et les problématiques liées à l'occupation du sol.

La figure 7 donne un aperçu du type de représentation cartographique obtenue suite à la procédure de désagrégation spatiale des sorties d'AROPAj (cf. section 2.3). Elle rend compte de la répartition des pratiques d'irrigation au niveau de la France entière et restitue les ordres de grandeur des quantités d'eau en jeu. Il convient de rappeler que ces valeurs symbolisent une demande en eau dans un contexte de maximisation de la marge brute agricole et n'apportent en rien une indication sur l'état de la disponibilité de la ressource. Les quantités d'eau d'irrigation variant de 25 (bleu clair) à $200 \mathrm{~m}^{3} / \mathrm{ha}$ (bleu foncé) se rapportent aux surfaces géographiques, étant entendu qu'AROPAj ne représente pas toute l'activité agricole du monde réel, que l'agriculture n'occupe qu'une partie de la surface (même si ont été exclus du calcul de probabilité spatiale les pixels dénués de toute activité agricole, l'activité agricole partage les terres avec d'autres occupations des terres telles que les forêts), et que l'irrigation ne concerne qu'une partie des cultures prises en compte par AROPAj.

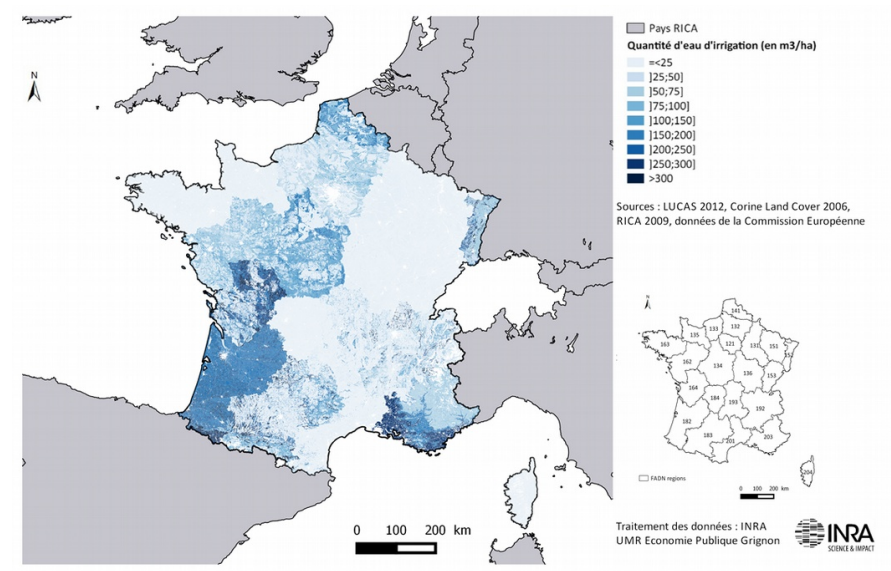

FIGURE 7. Demande en eau d'irrigation pour l'année 2009 en France 
La méthode de classification adoptée est celle des classes de seuils naturels. Globalement, l'année 2009 est marquée par de fortes disparités : la réponse des systèmes agricoles varie suivant les régions considérées. Les parties ouest (Aquitaine - 182 ; Centre - 134 ; Pays de la Loire - 162 ; Poitou-Charentes - 164 ; Midi-Pyrénées - 183) et sud-est de la France (Provence-Alpes-Côte d'Azur/PACA 203) se caractérisent par une forte demande en eau (supérieure à $150 \mathrm{~m}^{3} / \mathrm{ha}$ ). Ces valeurs semblent proches de celles renseignées par le ministère de l'agriculture pour 2009 (Eau France, 2012). Néanmoins, pour certaines régions, ces résultats sont fragiles en raison de l'existence de décalages entre données observées et estimations du modèle. Les lacunes en matière d'information sur les prélèvements en eau (concernant les stations de pompage) ou la non prise en compte par AROPAj d'activités agricoles fortement irriguées caractéristiques de certaines régions (e.g. l'horticulture) sont autant d'éléments à prendre en compte avant toute interprétation des résultats.

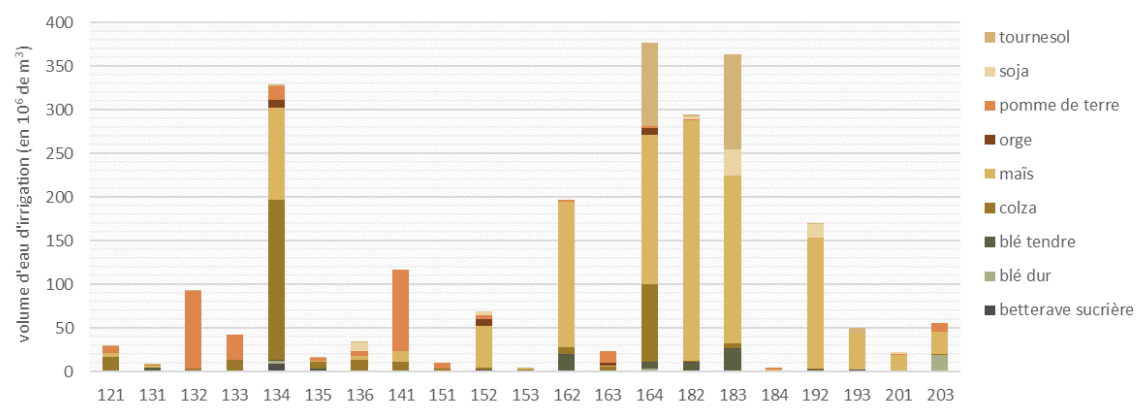

FIGURE 8. Etude régionale de la demande en eau d'irrigation par culture (2009)

Légende : 121 - Île-de-France ; 131 - Champagne-Ardenne ; 132 - Picardie ; 133 - Haute-Normandie ; 134 - Centre ; 135 - Basse Normandie ; 136 - Bourgogne ; 141 - Nord-Pas-de-Calais ; 151 - Lorraine ; 152 - Alsace ; 153 - FrancheComté ; 162 - Pays de la Loire ; 163 - Bretagne ; 164 - Poitou-Charentes ; 182 - Aquitaine ; 183 - Midi-Pyrénées ; 184 Limousin ; 192 - Rhône-Alpes ; 193 - Auvergne ; 201 - Languedoc-Roussillon ; 203 - Provence-Alpes-Côte d'Azur

En appui aux résultats précédents, la figure 8 récapitule les volumes d'eau requis par activité agricole et par région. Ici encore, de fortes hétérogénéités spatiales sont mises en évidence. Les 5 zones les plus irriguées citées précédemment, de même que l'Alsace, sont caractérisées par l'implantation du maïs, très consommateur d'eau d'irrigation. Les fortes demandes des régions Centre, Midi-Pyrénées et PoitouCharentes accompagnent des productions de tournesol et de colza fortement irrigués. La pomme de terre constitue l'un des systèmes culturaux les plus consommateurs au Nord de la France en régions Nord-Pas-de-Calais et Normandie. En revanche, il est important de noter que les quantités prélevées ne sont pas forcément corrélées aux surfaces irriguées du fait de la variabilité du facteur climat. De ce fait, les faibles volumes d'eau prélevés en Midi-Pyrénées exprimés dans la figure 8 ne révèlent pas nécessairement de faibles valeurs par hectare comme en atteste la carte précédente. 
Pour expliquer ces hétérogénéités, on peut s'appuyer sur la répartition des surfaces culturales entre régions fortement et faiblement irriguées (figure 9). Comme indiqué précédemment, maïs et blé dur en PACA, et tournesol en Poitou-Charentes et Midi-Pyrénées sont largement répandus dans les zones les plus irriguées. Parallèlement, les régions telles que le Limousin, l'Auvergne ou la Lorraine, moins consommatrices (figure 8), se caractérisent par la présence de prairies permanentes et de blé tendre non irrigué. Plus généralement, blé tendre, orge, colza et betterave sont majoritairement implantées dans le Nord de la France tandis que les cultures de blé dur et de tournesol sont plutôt présentes dans le Sud. Aller plus loin dans l'analyse demanderait une approche spatiale régionale plus fine du facteur climat, en particulier de la variable pluviométrique, en relation avec le choix de l'assolement et la répartition disparate des systèmes irrigués.

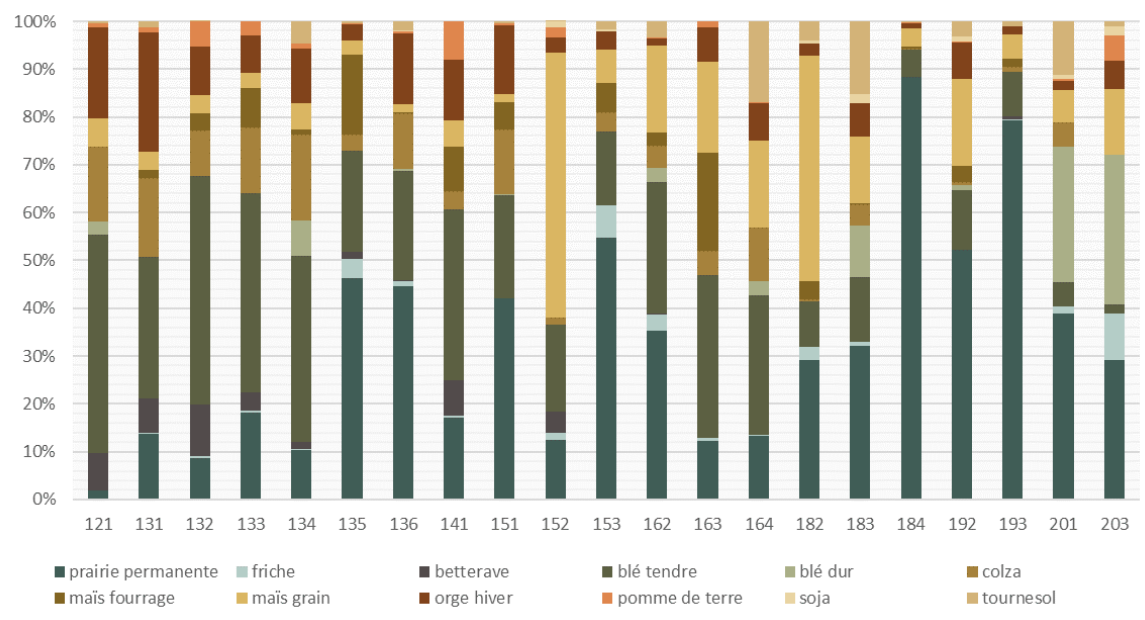

FIGURE 9. Répartition des surfaces (en \%) par culture et par région française

Légende : 121 - Île-de-France ; 131 - Champagne-Ardenne ; 132 - Picardie ; 133 - Haute-Normandie ; 134 - Centre ; 135 - Basse Normandie ; 136 - Bourgogne ; 141 - Nord-Pas-de-Calais ; 151 - Lorraine ; 152 - Alsace ; 153 - FrancheComté ; 162 - Pays de la Loire ; 163 - Bretagne ; 164 - Poitou-Charentes ; 182 - Aquitaine ; 183 - Midi-Pyrénées ; 184 -

Limousin ; 192 - Rhône-Alpes ; 193 - Auvergne ; 201 - Languedoc-Roussillon ; 203 - Provence-Alpes-Côte d'Azur

Comme spécifié précédemment, la culture de maïs constitue le système agricole le plus consommateur d'eau (figure 8). Cette activité en Poitou-Charentes combine une surface importante et une forte demande par hectare irrigué. Le focus sur les surfaces irriguées en maïs dans cette région permet donc une application illustrative intéressante (figure 10). Nous traitons un exemple d'analyse spatiale à une échelle plus fine que ce que propose la carte de la figure 7 afin de souligner la flexibilité de la méthode développée. Les valeurs varient de 500 (jaune clair) à $2000 \mathrm{~m}^{2}$ (rouge foncé) par hectare géographique. Ici encore, la méthode de classification adoptée est celle des classes de seuils naturels. La carte révèle que la production étudiée est principalement concentrée au Nord de la région. Néanmoins, il apparaît que la localisation de cette dernière dépend de la topographie de la zone. Les valeurs les 
plus fortes sont localisées le long du réseau hydrographique du fleuve Charente et de ses différents affluents. Nous observons, pour finir, une correspondance avec les résultats obtenus précédemment concernant le volume d'eau requis (en $\mathrm{m}^{3} / \mathrm{ha}$ ) du Poitou-Charentes toutes activités confondues (figure 7. Dans ce cas précis, l'implantation du maïs irrigué régit la demande d'irrigation. L'existence d'artefacts ou de brusques basculements de valeurs d'un pixel à l'autre s'explique par l'emploi d'une grille climatique étendue de $25 \times 25 \mathrm{~km}$ lors de la procédure de spatialisation. Cette carte met aussi en relief l'intérêt qu'il y aurait à lisser la représentation spatiale.

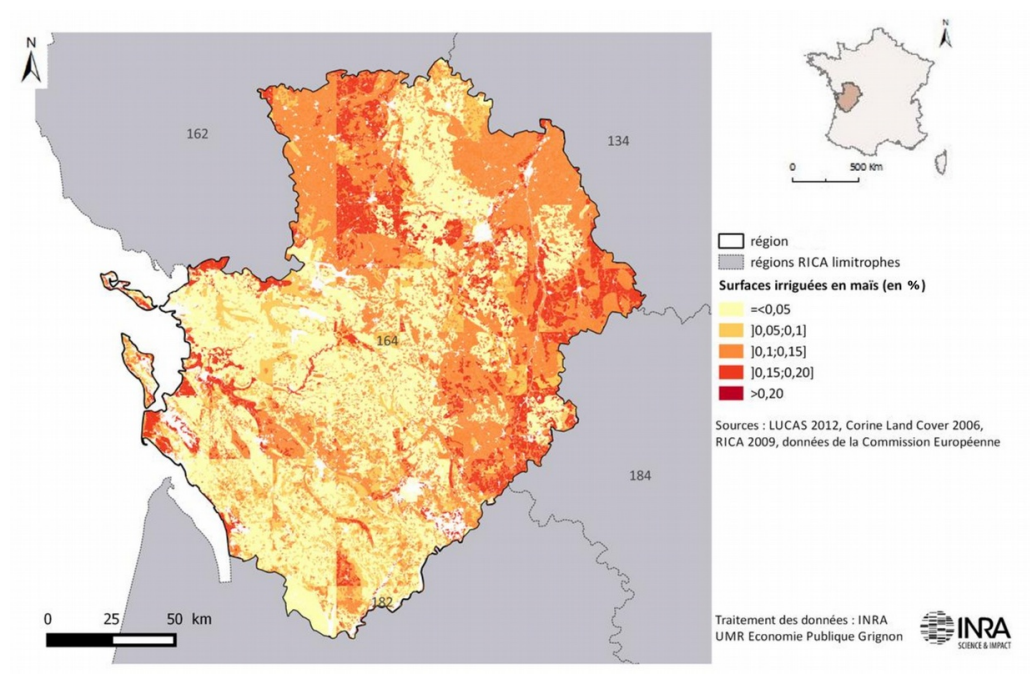

FIgURE 10. Part de surface pour la culture de maïs irrigué, Poitou-Charentes, 2009

\section{Conclusion et perspectives}

Compte tenu de la demande présente en eau d'irrigation, des pressions exercées sur la ressource en eau et des impacts probables du changement climatique sur la disponibilité en eau, le développement d'outils de modélisation appropriés apparaît indispensable à une gestion intégrée de la ressource. Dans cette optique, un modèle bioéconomique fondé sur le couplage de deux outils (AROPAj et STICS) et centré sur la réponse du rendement de culture aux intrants eau et azote est adopté. La méthode d'intégration de l'intrant « eau » développée par Humblot et al. (2017) est étendue. L'objectif est l'estimation de la demande d'irrigation d'une grande diversité de systèmes agricoles, dans un contexte de maximisation de leur marge brute, indépendamment d'une offre par ailleurs potentiellement limitante. De nombreuses variables, issues du traitement de multiples simulations, sont obtenues en sortie d'AROPAj à différentes échelles (exploitation agricole, bassin versant et/ou région). L'un des points forts de la méthode est la combinaison de ces simulations avec un module de spatialisation à haute résolution des résultats du 
modèle bioéconomique. La mobilisation de données physiques et de méthodes d'économétrie spatiale contribue à la désagrégation spatiale des résultats d'AROPAj à une échelle fine, différenciée par région et adaptée au contexte géographique et à la diversité des systèmes d'exploitation agricole.

La démarche initialement développée par Chakir (2009) et Cantelaube et al. (2012) pour une version antérieure d'AROPAj est étendue afin d'intégrer l'irrigation. Dans le champ des applications possibles, une attention particulière est accordée à l'étude de la demande en eau agricole en 2009, avec l'objectif d'illustrer la diversité et la richesse des sorties du modèle. La réalisation de représentations cartographiques à l'échelle de la France illustre la capacité de la méthode à saisir la diversité régionale des systèmes agricoles. Cette analyse spatiale met en évidence l'existence et la diversité des pratiques d'irrigation selon les régions, avec une irrigation principalement concentrée dans les parties ouest et sud-est de la France, pour ne s'en tenir qu'aux productions agricoles représentées par le modèle AROPAj. La demande forte qui caractérise ces zones traduit l'existence de cultures consommatrices d'eau d'irrigation (maïs, tournesol, colza, ...). Comme attendu, les régions moins consommatrices se définissent par la présence de prairie permanente et de blé tendre non irrigué.

Un focus sur les surfaces irriguées en maïs dans la région Poitou-Charentes constitue une illustration du type de traitements possibles à une échelle plus fine. L'une des perspectives offertes par la méthode que nous avons développée est d'étendre la démarche engagée à l'échelle européenne en intégrant le facteur climat et en étudiant l'impact de différentes politiques de régulation. Ce travail est rendu possible par un effort important accordé à l'automatisation des calculs dans les phases des processus d'estimation des paramètres et d'exploitation des résultats. Des analyses sont en cours de réalisation pour différents scénarios de projection climatique à l'échelle de la France. Le couplage AROPAj-STICS et la méthode de localisation probabiliste des agents économiques représentés par le modèle vont permettre d'avancer dans l'étude de l'adaptation des systèmes de production aux changements climatiques ou économiques annoncés.

\section{Bibliographie}

Brisson N., Gary C., Justes E., Roche R., Mary B., Ripoche D., Zimmer D., Sierra J., Bertuzzi P., Burger P., Bussière F., Cabidoche, Y. M., Cellier P., Debaeke P., Gaudillère J. P., Hénault C., Maraux F., Seguin B., Sinoquet, H. (2003). An overview of the crop model stics. European Journal of Agronomy, 18(3-4), 309-332.

Cantelaube P., Carles M. (2014). Le registre parcellaire graphique: des données géographiques pour décrire la couverture du sol agricole. Le Cahier des Techniques de l'INRA, (N. Spécial GéoExpé), pp.58-64.

https://www6.inra.fr/cahier_des techniques/content/download/3813/34098/version/2/file/ 12 CH2 CANTELAUBE registre parcellaire.pdf

Cantelaube P., Jayet P. A., Carré F., Bamps C., Zakharov P. (2012). Geographical downscaling of outputs provided by an economic farm model calibrated at the regional level. Land Use Policy, 29(1), 35-44. 
Chakir R. (2009). Spatial Downscaling of Agricultural Land-Use Data: An Econometric Approach Using Cross Entropy. Land Economics, 85(2), 238-251.

Cortignani R., Severini S. (2009). Modeling farm-level adoption of deficit irrigation using Positive Mathematical Programming. Agricultural Water Management, 96(12), 17851791.

Déqué, M., Dreveton, C., Braun, A., \& Cariolle, D. (1994). The ARPEGE/IFS atmosphere model: a contribution to the French community climate modelling. Climate Dynamics, 10(4-5), 249-266.

Eaufrance - Les prélèvements en eau en 2009 et leurs évolutions depuis 10 ans http://www.eaufrance.fr/ressources/documents/chiffres-statistiques-les

Godard C., Roger-Estrade J., Jayet P. A., Brisson N., Le Bas C. (2008). Use of available information at a European level to construct crop nitrogen response curves for the regions of the EU. Agricultural Systems, 97(1-2), 68-82.

Graveline N., Mérel P. (2014). Intensive and extensive margin adjustments to water scarcity in France's Cereal Belt. European Review of Agricultural Economics, 41(5), 707-743.

Humblot P., Jayet P.-A., Petsakos A. (2017). Farm-level bio-economic modeling of water and nitrogen use: Calibrating yield response functions with limited data. Agricultural Systems, $151,47-60$.

Janssen S. et van Ittersum M. K. (2007). Assessing farm innovations and responses to policies: are view of bio-economic farm models. AgriculturalSystems, 94 : 622-636.

Jayet P. A. et Petsakos A. (2013). Evaluating the efficiency of a uniform N-input tax under different policy scenarios at different scales. Environmental Modeling and Assessment, $18(1): 57-72$

Kampas A., Petsakos A., Rozakis S. (2012). Price induced irrigation water saving: Unraveling conflicts and synergies between European agricultural and water policies for a Greek Water District. Agricultural Systems, 113, 28-38.

Keating B. et Thorburn P. (2016), Modelling crops and cropping systems - evolving purpose, practice and prospects, International Crop Modelling Symposium, Berlin mars 2016, https://communications.ext.zalf.de/sites/crop-modelling/PDFDocuments/ 12 keating ppt keynote.pdf

Larson D. M., Helfand G.E. et House B. W. (1996) Second-Best Tax Policies to Reduce Non point Source Pollution. American Journal of Agricultural Economics, 78(4) : 1108- 1117

Leclère D., Jayet P.-A., De Noblet-Ducoudré N. (2013). Farm-level Autonomous Adaptation of European Agricultural Supply to Climate Change. Ecological Economics, 87, 1-14.

Medellín-Azuara J., Howitt R. E. et Harou J. J. (2012). Predicting farmer responses to water pricing, rationing and subsidies assuming profit maximizing investment in irrigation technology. Agricultural Water Management, 108 : 73-82. 